\title{
Identificación de las causas asociadas a las fallas del flujo de información para el apoyo logístico aguas arriba de la perforación offshore en Colombia
}

\author{
pags 111-124
}

Grupo de Investigación: Centro de Investigación y Desarrollo Empresarial CINDE

Línea de investigación: Nuevas tecnologías de perforación

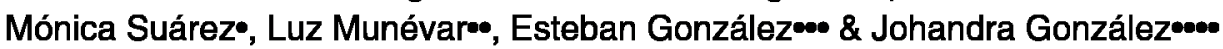

Recibido: 7 de mayo de 2015

\section{RESUMEN}

El intercambio de información se ha convertido en un factor clave para la colaboración a través de la cadena de suministro. A pesar de ello, la falta de sistemas de información, que articulen y soporten los procesos de abastecimiento, ocasiona demoras en las entregas programadas, y sobrecostos en los planes de proyecto. La identificación de causas asociadas a este problema, especificamente en el caso de las operaciones en la Costa Caribe Colombiana, se constituye en un avance que facilitará el desarrollo de una herramienta, en futuros trabajos, encaminada a optimizar el flujo de información en el área logística, para operaciones offshore en Colombia.

Palabras claves: Cadena de suministro, Colombia, Costa Afuera, Información, Sistema.

\section{ABSTRACT}

The exchange of information has become a key collaboration across the supply chain factor. However, the
Aceptado: 12 de junio de 2015

lack of information systems to articulate and support procurement processes, causes delays in scheduled deliveries and cost overruns in the project plans. The identification of causes related to this issue, specifically in the case of operations on the Colombian Caribbean coast, constitutes an advance that will facilitate the development of a tool in future studies aimed at optimizing the flow of information in logistics for offshore operations in Colombia.

Keywords: Supply Chain, Colombia, Offshore, Information, System.

\section{INTRODUCCIÓN}

La alta demanda de hidrocarburos en las últimas décadas, ha ocasionado que el hombre desarrolle nuevas técnicas para la obtención de energía, evitando que las actividades del mundo moderno se detengan. La perforación costa afuera, es una de esas innovadoras ideas que

\footnotetext{
- Docente investigadora y de programa Ingeniería Industrial. Fundación Universidad de América. monica.suarez@profesores.uamerica.edu.co

*- Estudiante coinvestigador Ingenieria Industrial. Fundación Universidad de América.

*. Estudiante coinvestigador Ingeniería Industrial. Fundación Universidad de América.

•... Estudiante coinvestigador Ingeniería Industrial. Fundación Universidad de América.
} 
llegaron a la industria del petróleo y gas, para quedarse y mejorar continuamente.

A pesar de que la perforación costa afuera es un poco más antigua de lo que se piensa, el desarrollo de actividades de apoyo, que facilitan el desarrollo de las operaciones en alta mar, es aún precario. Tal es el caso de la logística, una de las actividades más importantes, a la hora de dar soporte a las operaciones de exploración y producción de hidrocarburos. Cabe resaltar, la logística es concebida en diferentes escenarios, uno de ellos, y tal vez el más conocido, es el proceso de distribución posterior a la obtención de un producto. La cadena en la que se centra esta investigación, es por el contrario la denominada "aguas arriba", en donde el interés del abastecimiento se centra en satisfacer las necesidades de un centro de operaciones, como es el caso de las plataformas de perforación ubicadas mar adentro. Adicionalmente, es importante resaltar que la logística abarca tres grandes flujos: 1. el flujo principal de materiales y dos grandes flujos asociados a este movimiento de recursos. 2. el flujo de información y 3 . El flujo de dinero. En esta investigación, el interés principal es el manejo y gestión del flujo de información, el cual puede ser considerado como elemento clave, a la hora de conseguir el éxito en las operaciones diarias de cualquier organización.

En nuestro país es escaza la experiencia en perforación costa afuera, razón por la cual los avances desarrollados, pueden fácilmente constituir un gran adelanto en la actualidad, en lo que respecta al desarrollo de nuevos campos de producción de hidrocarburos. En el campo de la logística, realizar un diagnóstico de las condiciones actuales, en cuanto a flujo de información, se refiere a establecer parámetros para una transmisión, eficaz y eficiente de información. Puede, de ser implementado, resultar en la reducción de aumentos de presupuesto y retrasos en el cronograma de operaciones.

La identificación de las causas asociadas al problema de las fallas, en el flujo de información del área logística, para operaciones de explora- ción y producción offshore en Colombia, es el paso inicial para establecer técnicas, e incluso crear herramientas, que faciliten el proceso de transmisión de información, a lo largo de la cadena de suministro.

\section{MÉTODO}

Para desarrollar la identificación de las causas, asociadas al problema de investigación, se especificaron dos etapas principales. Cada una está asociada a actividades particulares, de la siguiente manera:

- Etapa exploratoria: recolección de información que permita definir un marco referencial que ubique al objeto de investigación dentro de límites teóricos, conceptuales, históricos $y$ legales.

- Etapa de diagnóstico: caracterización de las condiciones actuales de la operación logística offshore. Obtención de información a través de fuentes de información primaria y secundaria que permitan identificar las principales causas asociadas al problema de investigación. El proceso de diagnóstico se realizó con ayuda de herramientas como el Diagrama de Ishikawa, la Matriz Vester, entrevistas estructuradas, método Delphi y análisis de contexto y divergencia.

El objetivo principal del desarrollo de estas etapas es identificar las causas asociadas a las fallas del flujo de información para el apoyo logístico aguas arriba de la perforación offshore en Colombia a partir de fuentes de información secundaria, para luego validarlas a través de la aplicación de una entrevista estructura a expertos en logistica para operaciones de proyectos onshore y offshore en Colombia, el Golfo de México, Brasil y el Mar del Norte.

\section{Fundamentos teóricos}

La cadena de suministro en la industria del petróleo y gas, se divide en tres grandes seg- 
LÍNEA DE INVESTIGACION: NUEVAS TECNOLOGÍAS DE PERFORACIÓN

mentos: la cadena de abastecimiento upstream, la cadena midstream y la cadena de abastecimiento downstream. La cadena de suministro upstream se encarga de la adquisición de petróleo crudo, es decir, abarca todas las operaciones de exploración, previsión, producción y gestión de la logística para la entrega del petróleo. La cadena de suministro midstream se encarga de la entrega de petróleo crudo a las refinerias, así como de su procesamiento en subproductos. Finalmente, la cadena de suministro downstream, comienza a la salida de las refinerías, donde el crudo es distribuido y transportado a los puntos de venta (Hussain, Assavapokee \& Khumawala, 2006).

En la Figura 1 se puede observar cada uno de los segmentos de la cadena de abastecimiento de la industria del petróleo. La presente investigación está definida únicamente, dentro del segmento upstream de la cadena de suministro del petróleo. Especificamente para la etapa de exploración, en esta medida, se debe entender como cliente final a la plataforma o unidad de perforación costa afuera. Es decir, aquella que realiza requerimientos en orden de cumplir con la ejecución de sus operaciones.

La logística offshore, de manera más particular, considera la planificación, implementación y control del flujo, disponibilidad y almacenamiento de productos, insumos, requerimientos minimos del muelle, como son los equipos para cargas, línea de fluidos, la seguridad física del personal, el área de almacenamiento (abierta o cerrada), el transporte de la base de operaciones a la plataforma costa afuera, los planes de contingencia, y los procesos legales necesarios, para desarrollar las operaciones. Esto con el propósito de satisfacer, en el momento correcto, lugar preciso y forma adecuada, los requerimientos de la plataforma ubicada costa afuera.

En la Figura 2 se puede observar la cadena de suministro para el apoyo a la operación offshore. En esta cadena se debe considerar el flujo de materiales, de información y de capital. Adicionalmente, es importante definir la operación como un gran sistema, donde las entradas están asociadas a los requerimientos; la operación como el despliegue para cumplir con el abastecimiento; $y$ las salidas, como el resultado de cumplir con las solicitudes hechas a la entrada del sistema.

Figura 1. Segmentos de la cadena de suministro del petróleo y gas.

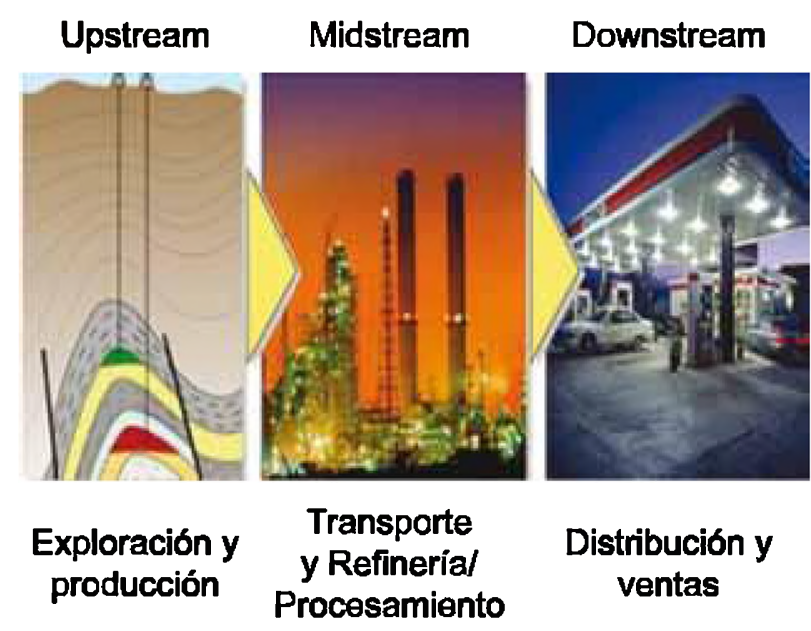

Fuente: Petroleum Online. (Recuperado 2014, junio).

Figura 2. Cadena de suministro para una instalación off-shore.

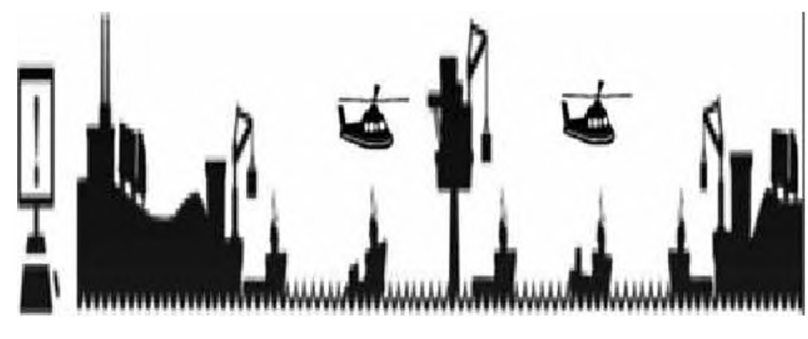

Procurement Supply Base actlvity Traneport Load/unload Transport Roverse loglettcos

Fuente: Vedde, N. \& Sagbakken, (2006)

Desde el punto de vista país es preciso indicar que, en la actualidad, Colombia ha iniciado una fase de expansión de su industria petrolera, con inversiones importantes en infraestructura y tecnología. Pero aún hay fallas en factores claves, como el caso de la gestión de la información y, aunque la producción en plataformas costa fuera está en una fase inicial creciente, 
ya existen inconvenientes que, incluso, han sido previstos en antecedentes de compañias del sector en otros países.

La etapa de diagnóstico permitió la obtención de datos e información necesaria, para identificar las principales fallas en el intercambio de información, y definir las variables principales involucradas en el proceso logístico offshore. A través del diagnóstico de las prácticas y procesos utilizados, en el flujo de información logístico, se pretendió determinar cuáles son las causas con mayor incidencia dentro de las fallas, en el intercambio de información.

Gracias a una revisión bibliográfica continua, y basada en diferentes escenarios a nivel mundial, se evidencia la existencia de fallas en los flujos de información, que permiten el suministro de recursos $y$, por ende, responder a las necesidades de las plataformas de perforación costa fuera (Favilla, Claessens, Mello, Flach \& IBM, 2012). Entre algunas de las causas, se puede mencionar la infraestructura insuficiente para la adecuación de herramientas, que facilitan el intercambio de la información. Esto se ve reflejado en la existencia de diversas aplicaciones, usadas para su gestión, ocasionando que no se logre transmitir y conocer en tiempo real. Desde una perspectiva diferente, la normatividad nacional incipiente, para el intercambio de información, puede generar rupturas y distorsión en el flujo informativo.

Por otro lado, es importante resaltar, desde el punto de vista del talento humano, y en especial para el caso colombiano, las personas y las empresas mantienen un enfoque tradicionalista para compartir información. Éste se refleja en la falta de cooperación, que impide que los riesgos y recompensas se asuman de manera compartida, entre los actores involucrados en la cadena de suministro. Por ende, cada recurso persigue su propio objetivo, y no el general, que es la idea preponderante en la teoría de la cadena de abastecimiento.

Igualmente, es preciso argumentar una causa importante que impide el correcto flujo de información, y por ende, de bienes, aparte del desconocimiento y falta de organización de las estructuras existentes. Éste es el elevado costo de implementación de herramientas de gestión de información, que además conlleva a cambios internos en la organización, en donde el valor se centra en lo colectivo y no en lo particular. Así también, las múltiples capacitaciones en las que se verían involucrados los agentes interesados en las operaciones.

Es preciso indicar, adicionalmente, que la proliferación de acciones correctivas puede causar un traumatismo importante, dentro de la estructura de la organización, limitando las acciones preventivas y de planeación. Al no tener cabida, conllevarían a una incertidumbre en las operaciones pertinentes a la cadena de suministro, y a limitaciones en la capacidad de la empresa, para el desarrollo de proyectos que la puedan beneficiar a largo plazo.

Considerando la integración limitada de los actores de la cadena de suministro, así como la falta de una relación comercial estrecha, enmarcada por suministros esporádicos, o bajo condiciones poco estables, pueden ocasionarse conflictos entre los proveedores y las plataformas costa fuera. Esto, debido a que las bases de operación, que funcionan como agentes intermediarios, no podrían integrar plenamente los procesos ni las soluciones, dejando plataformas insatisfechas, obligándolas a reprogramar sus actividades sin reparar en los sobrecostos generados por tales situaciones.

De igual manera, la ausencia de un canal robusto de integración, y de gestión de la información entre las partes involucradas, para suplir un requerimiento de una plataforma costa fuera, ha reflejado que la base en tierra no cumple su función de apoyo. Por el contrario, se convierte en la figura antagónica, al carecer de habilidad y herramientas para acoplar los agentes de la cadena de suministro, y hacer que funcionen en armonía con las operaciones, convirtiéndose así en un punto neurálgico de intervención, y así evitar afectaciones en la 
LÍNEA DE INVESTIGACIÓN: NUEVAS TECNOLOGÍAS DE PERFORACIÓN

estructura financiera y corporativa de la organización.

La identificación de las causas fue realizada con ayuda de la lluvia de ideas o brainstorming. Esta técnica posibilita la creatividad y se basa en una discusión grupal, generada a partir de una pregunta planteada por el moderador (Winter, 2000). La lluvia de ideas tiene un propósito exploratorio, y esta característica es útil en las primeras etapas de una investigación. En la revisión se identifican semejanzas, para ordenar y clasificar las ideas por temas, parte que corresponde a un análisis de contenido (Vázquez Navarrete, Ferreira Da Silva, Mogollón Pérez, \& Sanmamed Santos, 2005). Las caracteristicas de esta técnica facilitan la creación de un listado de posibles causas del problema de investigación, favoreciendo el desarrollo de las etapas posteriores $y$, de manera generalizada, el avance del diagnóstico. Una vez se han listado las causas, se clasifican de manera ordenada a través del diagrama de Ishikawa, específicamente por el método de las $6 \mathrm{M}$ y el de estratificación. Es decir, se utilizaron dos metodologías que definen el método seleccionado, como diagrama de Ishikawa mixto. Un diagrama causa-efecto que agrupa dos metodologías, mitiga las desventajas de cada uno, y permite aprovechar las características, al favorecer el análisis planteado, en esta parte del diagnóstico. El diagrama resultante se puede observar en la Figura 3.

El diagrama de Ishikawa permitió distinguir 38 causas. Desde una perspectiva ambigua no se habrían identificado a plenitud. La postulación de las causas a través de la lluvia de ideas, sin juzgar por su incidencia o repetición, permitieron un conglomerado importante de ideas a analizar, las cuales, una vez concretadas, facilitaron la conformación de las categorías, hasta obtener el diagrama de Ishikawa. Bajo éste, se fundamentaron las causas de interés y de mayor repercusión sobre el problema identificado, respecto de las fallas en el flujo de la información. Aunque la postulación de causas fue arbitraria en aras de una mayor profundidad en el análisis, es preciso resaltar que cada postulación cuenta con un fundamento teó- rico o probatorio a través de conferencias, reuniones con expertos en el área de estudio, tesis de maestría y artículos de investigación, entre otros.

Algunas de las causas y su respectiva fuente, se pueden identificar a continuación:

- Canales de transmisión de información inadecuados. (Fontana, Papa, Marte, Yoshioka, \& Sakurai, 2014)

- Procesos extensos en fases de solicitudaprobación, aprobación-adquisición, adquisición-despacho. (Favilla et al., 2012)

- Falta de retroalimentación de la información. (Chima \& Hills, 2007)

- Ausencia en la definición de responsables y usuarios de la información. (Karalkova \& Kilcheuskaya, 2011)

- Incompatibilidad en los sistemas gestión de información. (Chima \& Hills, 2007)

- Emisiones por subutilización de recursos de transporte. (Kukareko, 2013)

- Suministro de requerimientos sin identificación ni etiquetas. (Rajoo \& Esso Exploration Angola Ltd., 2006).

- Falta de capacitación.(Karalkova \& Kilcheuskaya, 2011; Rajoo \& Esso Exploration Angola Ltd., 2006)

- Personal calificado limitado. (Favilla et al., 2012)

- Desconocimiento de la legislación para perforación offshore. (Rajoo \& Esso Exploration Angola Ltd., 2006; Vizcaíno, 2012)

La clasificación de las causas mediante el diagrama de Ishikawa, permitió dar paso a la priorización de variables a través de la matriz Vester. La matriz Vester busca, por medio de la relación de variables, reconocer cuáles de estas son criticas, activas, pasivas e indiferentes. Esta herramienta fue desarrollada como una técnica, para facilitar la identificación y relación de las causas y consecuencias, de una situación 


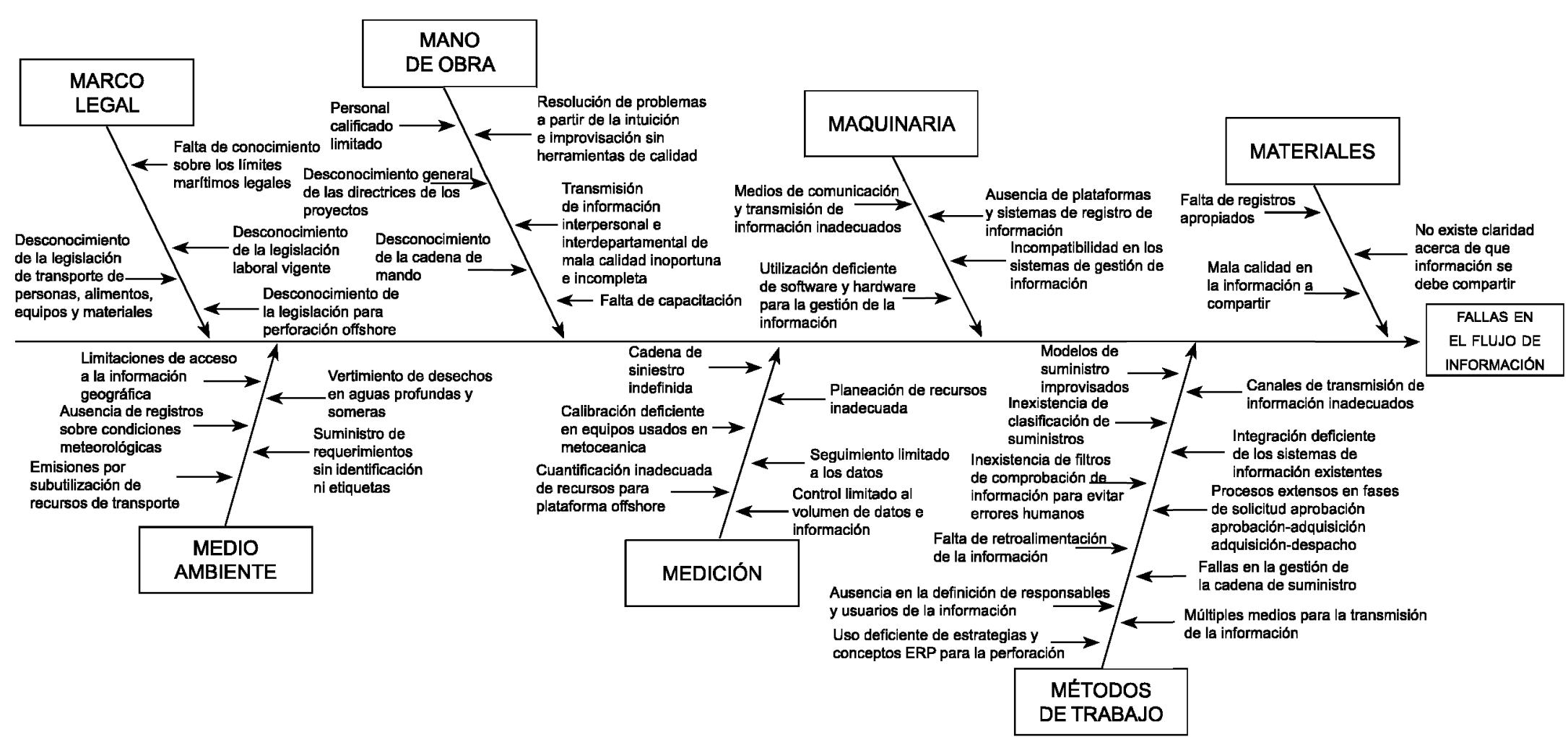

Fuente: Los Autores. 
problemática, de manera que se pueda determinar cuáles son las causas o problemas más críticos, cuáles son sus consecuencias, y fijar planes $\mathrm{o}$ acciones correctivas, así como preventivas y de control (Holguin Aguirre, Bonilla Luque, Pupo Gómez, \& Lezaca Sánchez, 2013).

La metodología de la matriz busca evaluar el grado de causalidad ( 1 a 3 ), que tiene la causa 1 sobre la causa 2 , la causa 3 y, de manera sucesiva, hasta llegar a la causa 38. En orden de facilitar la comprensión del proceso de construcción de la matriz, el siguiente es un ejemplo del tipo de preguntas utilizadas para realizar la calificación de las variables: ¿Conlleva la integración deficiente de los sistemas de información existentes, a procesos extensos en fases de solicitud-aprobación, aprobación-adquisición, adquisición-despacho? La incidencia de este causa es alta por lo que su puntaje será de tres (3).

El resultado de la aplicación de la matriz de Vester a las causas, se puede observar en la Figura 4. En la fase posterior del diagnóstico, se validaron las causas a través de entrevistas estructuradas a expertos y de esta manera se concluye el objetivo principal de ésta etapa de la investigación en donde se definen las causas más incidentes en el problema de investigación identificado.

A pesar de inicialmente considerar, como las causas más significativas, y a criterio de los investigadores, a aquellas ubicadas en el cuadrante de activas y críticas, algunas causas importantes pudieron haber quedado clasificadas dentro de las pasivas e indiferentes. Por ello es importante realizar una revisión de diferente carácter, a través de la consulta de fuentes de información primaria.

En esta medida, la herramienta que permitió validar la incidencia e importancia de las causas, fue un cuestionario facilitador del desarrollo de la entrevista a expertos, bajo los parámetros de la Teoría de Respuesta al Ítem (TRI) y las Escalas de Likert.
A manera de ejemplo, en el caso de la Teoría de Respuesta al litem, una de las preguntas diseñadas corresponde a la opción de calificación en escala de 1 a 3 . El objetivo de esta pregunta es conocer la incidencia de las alternativas, relacionadas al factor humano, en las fallas de los procesos de las operaciones off-shore. Su encabezado y respectivas opciones se exponen a continuación:

- Al ser las personas parte activa y determinante en la actividad logística, califique de 1 a 3 la incidencia de los siguientes factores en las fallas de los procesos, de las operaciones off-shore. (Siendo 1 poco incidente, 2 medianamente incidente y 3 muy incidente).

- Falta de capacitación. $-^{1} \_^{2} \_^{3}$

- Personal calificado limitado. $\ldots$ ${ }^{2}-3$

- Desconocimiento general de las directrices de los proyectos.

- $-^{1}-^{2}{ }^{3}$

- Desconocimiento de la cadena de mando. $\quad-1{ }^{1}{ }^{2}{ }^{3}$

Por otro lado, en el caso de las Escalas de Likert, el siguiente ejemplo está asociado a corroborar, si la utilización deficiente de software y hardware incide en el tiempo de respuesta, a los requerimientos de la plataforma. Su encabezado y respectivas opciones se exponen a continuación:

- El tiempo de respuesta a las necesidades de la plataforma, depende en gran proporción del medio de comunicación por el que se transmiten las mismas.

- Definitivamente sí.

- Indeciso.

- Definitivamente no. 


\section{Figura 4. Matriz de Vester.}

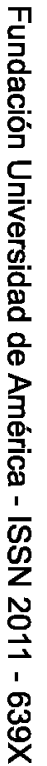

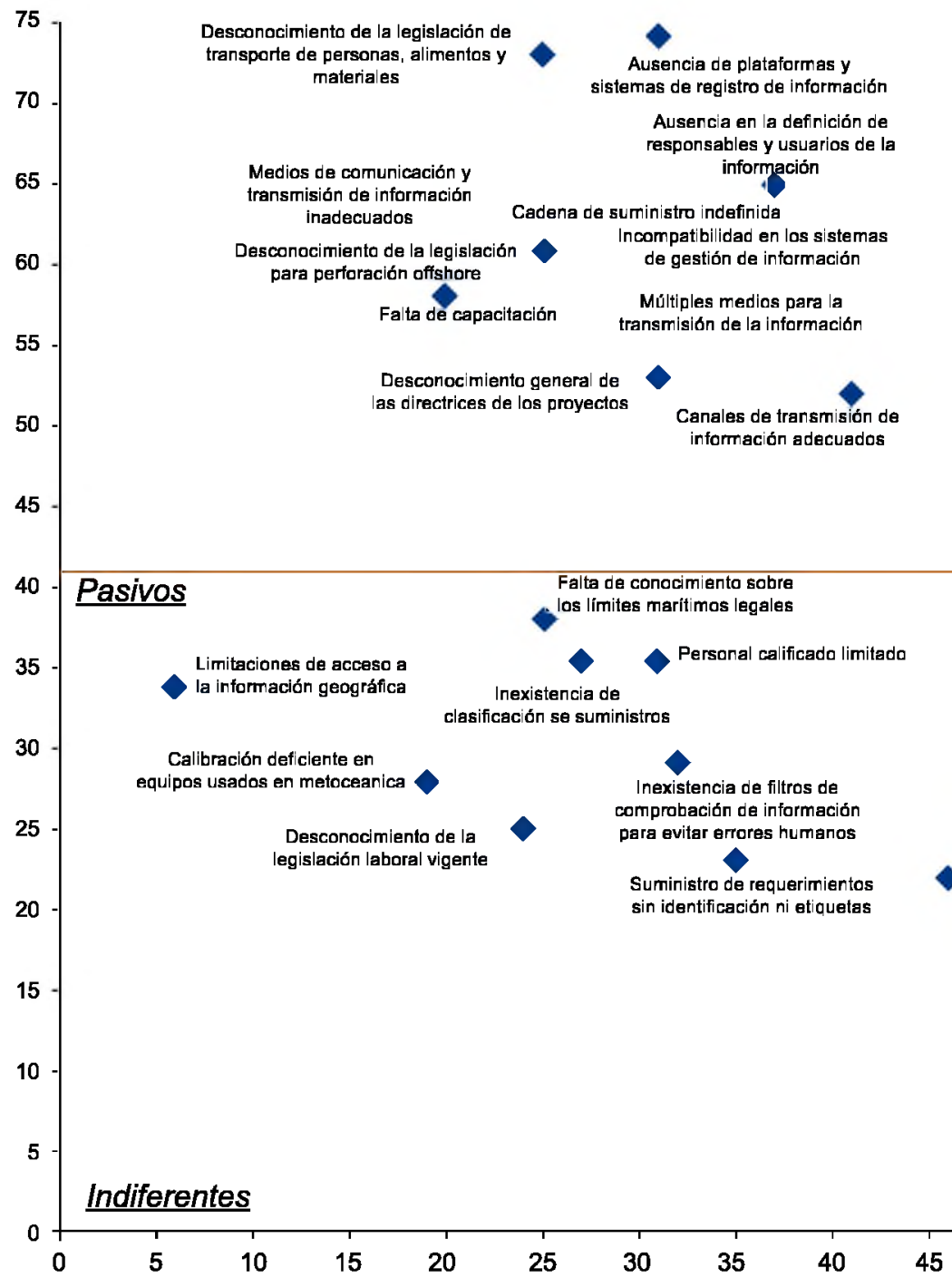

Fuente: Los Autores. 
Utilización deficiente de software y hardware para la gestión de la información

Integración deficiente de los

sistemas de información existentes
Desconocimiento de la

cadena de manco

Falta de retroalimentación de la información

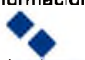

Uso deficiente de estrateglas y

conceptos ERP para la perforación

Modelos de suministro improvisados
Seguimiento limitada a la calidad de los dafos

\section{No existe claridad acerca de que}

información se debe compartir

Planeación de recursos inadecuada

Mala calidad en la

información a compartir

Transmisión de

información interpersonal e

intercepartamental de mala

calldad, inoportuna e incompleta

Cuantificación inadecuada de recursos para plataforma offshore
Procesos extersos en fases de solicitud-aprobación, aprobaciónadquisición, adquisición-cespacho

$$
\checkmark
$$

Resolución de problemas a partir de la intuición e improvisación sin herramientas ce calidad

Emisiones por subutilización

Activos

de recursos de transporte

$\begin{array}{llllllll}50 & 55 & 60 & 65 & 70 & 75 & 80 & 85\end{array}$


LÍNEA DE INVESTIGACIÓN: NUEVAS TECNOLOGÍAS DE PERFORACIÓN

La entrevista cumplió con una validación inicial, con el objetivo de identificar fallas en la estructura, y el correcto entendimiento de las preguntas. Una vez se consideraron las observaciones de los validadores, y se estructuraron nuevamente las preguntas con las recomendaciones, dadas en este proceso, el paso siguiente consistió en contactar, a través de la técnica bola de nieve, a los expertos que cumplían con las características definidas dentro del perfil para la investigación.

Los expertos contactados fueron 6 profesionales, con experiencia en el área logística de proyectos, realizados en alta mar, del sector de hidrocarburos en Colombia. Es importante resaltar que, dentro del grupo contactado, se reúne experiencia de proyectos onshore y offshore en Colombia, el Golfo de México, Brasil y el Mar del Norte. En el caso particular de Colombia, es importante resaltar la experiencia de los expertos en el desarrollo del proyecto Mapalé, en la Costa Caribe Colombiana.

A partir de diferentes análisis realizados (contexto y divergencia), una vez organizada la información a través de las entrevistas, se concluye que ésta es la mejor herramienta para conocer e identificar las falencias relacionadas con los flujos de información. Esto, al permitir un aporte de experiencias, conocimientos e incluso opiniones, que permiten fortalecer las bases, sobre las que se fundamentará la propuesta de respuesta a estas fallas, así como percibir puntos claves a observar en el momento de plantearlas.

Con el objetivo de incorporar el análisis realizado, previamente a través del uso de la matriz Vester y el diagrama de Ishikawa, se presenta el Cuadro 1, en el que se ejemplifica el caso de las preguntas 2 y 3 . Estas están en relación a la prioridad dada por la matriz versus la determinada a través de la consulta a expertos. Las causas con prioridad alta o media, se consideraron nodivergentes respecto a las causas identificadas como críticas o activas en la matriz de Vester. Se realizó el mismo tratamiento para las causas identificadas con prioridad baja, comparadas con las causas identificadas como indiferentes o pasivas en la matriz. Cualquier caso contrario se consideró como divergente.

Es importante profundizar en este punto, en el manejo dado a las causas, respecto del análisis de divergencia expuesto de manera ordenada en el cuadro anterior. Ejemplo de esto, el caso de la opción $\mathrm{C}$ de la pregunta 3 . La prioridad de la matriz se definió como Pasivo, mientras que a través del análisis de la entrevista estructurada, la prioridad de los expertos para la misma opción se definió como Alta, constituyendo una divergencia. La causa asociada a esta pregunta es la ausencia en la definición de responsables y usuarios de la información que, desde la perspectiva de los entrevistados, es uno de los factores determinantes, a la hora de estructurar un proceso de comunicación exitoso en el área logística. En esta medida, en algunas de las causas, como es el del caso expuesto, la opinión de la fuente de consulta primaria prima sobre la calificación realizada con ayuda de la matriz Vester. De esta manera, a partir de los resultados descritos en el Cuadro 1, se definen las causas asociadas a las fallas del flujo de información, para el apoyo logístico aguas arriba de la perforación offshore en Colombia.

Una vez se han realizado los análisis correspondientes a cada una de las diferentes tipologías utilizadas, en las preguntas del cuestionario, se definen las causas más significativas. Éstas pueden observarse en el cuadro 2 , con su respectiva relación en la clasificación del diagrama de Ishikawa.

\section{CONCLUSIONES}

A partir del análisis de causas más representativas, en las fallas del proceso de transmisión de información en el área logística, se identificó la importancia del manejo de los datos, tanto en la plataforma, como en el shore-base encargado de abastecerla. En la actualidad, la información requerida se almacena en diferentes sistemas y "pertenece" a diferentes miembros de la cadena 
de suministro. Adicionalmente, las personas en el shore-base deben acceder a diferentes aplicaciones, e ir a variados sitios web, para recopilar información de forma manual, y luego tratar de encontrarle sentido para trabajar con ella. En la mayoría de los casos, hay muy poco tiempo disponible para la toma de decisiones, y este proceso manual de obtención de información, ocasiona gran parte de los problemas logísticos.

Es importante mencionar que fue identificada la necesidad de realizar una validación, con ayuda de entrevistas, una vez terminada la primera fase del proceso de diagnóstico. Esto, a través de la consulta de fuentes secundarias, debido a algunas discrepancias, según criterio de los investigadores.

Adicionalmente, es importante resaltar, dentro del proceso de consulta a expertos, se rescataron experiencias que permitirán, en fases posteriores de la investigación, darle un mejor enfoque y orientación al diseño de una herramienta para mitigar las fallas identificadas en esta fase.
Algunas de estas experiencias incluyen, dentro de la operatividad, la contratación de expertos no especializados, la falta de directrices y mecanismos de comunicación eficaces, la falta de integración y la experiencia insuficiente a la hora de realizar proyectos offshore en el país. Dentro de la planeación, la falta de estructuras de red para la comunicación de requerimientos, la ausencia en la definición de responsables en manejo de equipos y usuarios de información, falta de protocolos y procedimientos para eventos extraordinarios o emergencias.

Finalmente, dentro del tópico de información los expertos resaltan los errores en el análisis de la información mete-oceánica, la falta de retroalimentación y de mecanismos para el registro y trazabilidad de la información, la limitación en el proceso de comunicación, por la diversidad de idiomas del personal que participa en los proyectos, y el rompimiento continuo de la cadena de información, que impide la respuesta eficiente ante eventos no planeados.

\section{Cuadro 1. Ejemplo conclusión relación de causas.}

\begin{tabular}{|c|c|c|c|c|c|}
\hline Pregunta & Opción & Causa & $\begin{array}{l}\text { Prioridad } \\
\text { Matriz Vester }\end{array}$ & $\begin{array}{l}\text { Prioridad } \\
\text { Expertos }\end{array}$ & Conclusión \\
\hline \multirow{5}{*}{2} & A & $\begin{array}{l}\text { Procesos extensos en fases de } \\
\text { solicitud-aprobación, aprobación- } \\
\text { adquisición, adquisición-despa- } \\
\text { cho. }\end{array}$ & Activo & Media & $\begin{array}{l}\text { No divergen- } \\
\text { cia }\end{array}$ \\
\hline & B & $\begin{array}{l}\text { Planeación de recursos inade- } \\
\text { cuada. }\end{array}$ & Crítico & Alta & $\begin{array}{l}\text { No divergen- } \\
\text { cia }\end{array}$ \\
\hline & C & $\begin{array}{l}\text { Ausencia de registros sobre con- } \\
\text { diciones meteorológicas. }\end{array}$ & Activo & Baja & Divergencia \\
\hline & D & $\begin{array}{l}\text { Transmisión de información inter- } \\
\text { personal e interdepartamental de } \\
\text { mala calidad, inoportuna e incom- } \\
\text { pleta. }\end{array}$ & Activo & Media & $\begin{array}{l}\text { No divergen- } \\
\text { cia }\end{array}$ \\
\hline & $E$ & $\begin{array}{l}\text { Desconocimiento de la legisla- } \\
\text { ción de transporte de personas, } \\
\text { alimentos, equipos y materiales. }\end{array}$ & Pasivo & Alta & Divergencia \\
\hline
\end{tabular}


LÍNEA DE INVESTIGACIÓN: NUEVAS TECNOLOGIAS DE PERFORACIÓN

\begin{tabular}{|c|c|c|c|c|c|}
\hline Pregunta & Opción & Causa & $\begin{array}{l}\text { Prioridad } \\
\text { Matriz Vester }\end{array}$ & $\begin{array}{l}\text { Prioridad } \\
\text { Expertos }\end{array}$ & Conclusión \\
\hline \multirow{11}{*}{3} & A & $\begin{array}{l}\text { Canales de transmisión de infor- } \\
\text { mación inadecuados. }\end{array}$ & Pasivo & Media & Divergencia \\
\hline & B & $\begin{array}{l}\text { Integración deficiente de los siste- } \\
\text { mas de información. }\end{array}$ & Crítico & Alta & $\begin{array}{l}\text { No } \\
\text { divergencia }\end{array}$ \\
\hline & $\mathrm{C}$ & $\begin{array}{l}\text { Ausencia en la definición de res- } \\
\text { ponsables y usuarios de la infor- } \\
\text { mación. }\end{array}$ & Pasivo & Alta & Divergencia \\
\hline & D & $\begin{array}{l}\text { Múltiples medios para la transmi- } \\
\text { sión de la información. }\end{array}$ & Pasivo & Media & Divergencia \\
\hline & $E$ & $\begin{array}{l}\text { Control limitado al volumen de da- } \\
\text { tos e información. }\end{array}$ & Crítico & Baja & Divergencia \\
\hline & $F$ & $\begin{array}{l}\text { Medios de comunicación y trans- } \\
\text { misión de información inadecua- } \\
\text { dos. }\end{array}$ & Pasivo & Media & Divergencia \\
\hline & G & $\begin{array}{l}\text { Ausencia de plataformas y siste- } \\
\text { mas de registro de información. }\end{array}$ & Pasivo & Alta & Divergencia \\
\hline & H & $\begin{array}{l}\text { No existe claridad acerca de qué } \\
\text { información se debe compartir. }\end{array}$ & Crítico & Alta & $\begin{array}{l}\text { No } \\
\text { divergencia }\end{array}$ \\
\hline & I & $\begin{array}{l}\text { Inexistencia de filtros de compro- } \\
\text { bación de información para evitar } \\
\text { errores humanos. }\end{array}$ & Indiferente & Baja & $\begin{array}{l}\text { No } \\
\text { divergencia }\end{array}$ \\
\hline & J & $\begin{array}{l}\text { Seguimiento limitado a la calidad } \\
\text { de los datos. }\end{array}$ & Crítico & Baja & Divergencia \\
\hline & K & $\begin{array}{l}\text { Mala calidad en la información a } \\
\text { compartir. }\end{array}$ & Activo & Alta & $\begin{array}{l}\text { No } \\
\text { divergencia }\end{array}$ \\
\hline
\end{tabular}

Fuente: Los Autores.

Cuadro 2. Causas más representativas.

\section{Causa}

Procesos extensos en fases de solicitud-aprobación, aprobación-adquisición, adquisicióndespacho.

Suministro de requerimientos sin identificación ni etiquetas.

Modelos de suministro improvisados.

Canales de transmisión de información inadecuados.

Métodos de

Integración deficiente de los sistemas de información.

Ausencia en la definición de responsables y usuarios de la información.

Múltiples medios para la transmisión de la información. 


\begin{tabular}{|c|c|}
\hline Causa & Clasificación \\
\hline Falta de capacitación. & \multirow{6}{*}{ Mano de Obra } \\
\hline Personal calificado limitado. & \\
\hline Desconocimiento general de las directrices de los proyectos. & \\
\hline Desconocimiento de la cadena de mando. & \\
\hline $\begin{array}{l}\text { Transmisión de información interpersonal e interdepartamental de mala calidad, inoportu- } \\
\text { na e incompleta. }\end{array}$ & \\
\hline $\begin{array}{l}\text { Resolución de problemas a partir de la intuición e improvisación sin herramientas de cali- } \\
\text { dad. }\end{array}$ & \\
\hline Utilización deficiente de software y hardware para la gestión de la información. & \multirow{7}{*}{$\begin{array}{l}\text { Maquinaría } \\
\text { (ERP) }\end{array}$} \\
\hline Incompatibilidad en los sistemas de gestión de información. & \\
\hline Medios de comunicación y transmisión de información inadecuados. & \\
\hline Ausencia de plataformas y sistemas de registro de información. & \\
\hline Inexistencia de filtros de comprobación de información para evitar errores humanos. & \\
\hline Uso deficiente de estrategias y conceptos ERP para la perforación. & \\
\hline Inexistencia de clasificación de suministros. & \\
\hline No existe claridad acerca de qué información se debe compartir. & \multirow{3}{*}{ Materiales } \\
\hline Mala calidad en la información a compartir. & \\
\hline Falta de registros apropiados. & \\
\hline Desconocimiento de los límites marítimos legales. & \multirow{3}{*}{ Marco Legal } \\
\hline Desconocimientos de la reglamentación ambiental. & \\
\hline $\begin{array}{l}\text { Desconocimiento de la legislación de transporte de personas, alimentos, equipos y mate- } \\
\text { riales. }\end{array}$ & \\
\hline Cuantificación inadecuada de recursos para plataforma offshore. & \multirow{3}{*}{ Medición } \\
\hline Planeación de recursos inadecuada. & \\
\hline Cadena de suministro indefinida. & \\
\hline Emisiones por subutilización de recursos de transporte. & \multirow[b]{2}{*}{ Medio Ambiente } \\
\hline $\begin{array}{l}\text { Ausencia de estandarización para el vertimiento de desechos en aguas profundas y so- } \\
\text { meras. }\end{array}$ & \\
\hline
\end{tabular}

Fuente: Los Autores.

El proceso de diagnóstico de las prácticas y procesos en el intercambio de información del área logística para operaciones offshore en Co- lombia, responde a una secuencia de actividades estructurada que se puede observar en la Figura 5. 
Figura 5. Secuencia proceso de diagnóstico

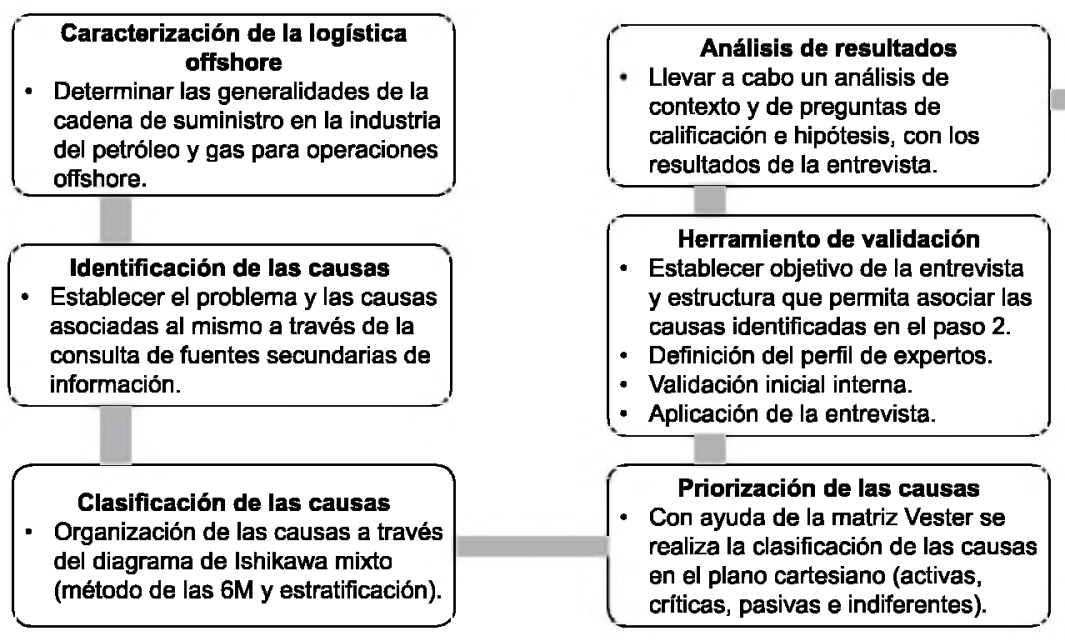

Fuente: Los Autores

\section{REFERENCIAS}

Chima, C. M., \& Hills, D. (2007). "Supply-Chain Management Issues in the Oil and Gas Industry". Journal of Business \& Economics Research, 5(6), 27-36.

Favilla, J. R., Claessens, D. A., Mello, U., Flach, B., \& IBM. (2012). "Achieving Excellence in Offshore Logistics". SPE.

Fontana, C. F., Papa, F., Marte, C. L., Yoshioka, L. R., \& Sakurai, C. A. (2014). "Intelligent Transportation System as a part of Seaport Terminal Management System". International Journal of Systems Applications, Engineering \& Development, 8.

Holguin Aguirre, M., Bonilla Luque, P., Pupo Gómez, A. A., \& Lezaca Sánchez, J. A. (2013). "Guía metodológica para la formulación de proyectos ambien- tales escolares". Consultado el 08 de Julio de, 2014, disponible en: http://www.unilibre.edu.co/ sga/images/stories/-pdfs/2013/ guiafinal.pdf

Hussain, R., Assavapokee, T., \& Khumawala, B. (2006). "Supply chain management in the petroleum industry: challenges and opportunities". Journal of Global Logistics \& Supply Chain Management, 1(2), 90-97.

Karalkova, A., \& Kilcheuskaya, K. (2011). "X2X: A new conceptual solution on the edge of information systems and supply chain management". Molde University College.

Kukareko, K. (2013). "Virtual warehousing in offshore oil and gas platforms" supply chain. Molde University College.

Petroleum Online. Recuperado 10 de junio de 2014, de http://www.petroleumonline.com/content/overview. asp?mod=8.

Rajoo, B. K., \& Esso Exploration Angola Ltd. (2006). "Development of the Kwanda Base Deepwater Logistics Shorebase" Offshore Technology Conference.

Vázquez Navarrete, M. L., Ferreira Da Silva, R., Mogollón Pérez, A., \& Sanmamed Santos, J. (2005). "Introducción a las técnicas cualitativas de investigación aplicadas en salud". (pp. 45-48). Barcelona: Servei de Publicacions Universitat Autónoma de Barcelona.

Vedde, Nikolai y Sagbakken, Oyvind. An analysis of the planning horizon for logistics activities at the Heidrun Offshore Installation. Supply Chain for an Offshore Installation Like HD (Nygard 2006). 2010. p.10. 
Vizcaíno, O. L. (2012). ecopetrol.com.co/especiales/ Winter, R. S. (2000). "Ma"El Mischief está en Carta- CartaPetrolera128/-explora- nual de trabajo en equipo" Edigena de Indias". Consulta- cion.htm do el 10 de Agosto de 2014. ciones Barcelona., pp. 19-21). Disponible en: http://www. Madrid. 